\title{
Beam-ion instability and its mitigation with feedback system
}

\author{
Chao Li๑," Saike Tian๑, Na Wang, and Haisheng Xu \\ Key Laboratory of Particle Acceleration Physics and Technology, Institute of High Energy Physics, \\ Chinese Academy of Sciences, 19(B) Yuquan Road, Beijing 100049, China
}

(Received 14 November 2019; accepted 29 June 2020; published 6 July 2020)

\begin{abstract}
The beam-ion interaction is one of the potential limitations of beam performance in ultralow-emittance electron light sources. Conventionally, the beam-ion instability is attributed to two effects: the ion-trapping effect and fast ion effect, which are usually studied individually emphasizing the beam performance in different timescales. However, in accelerators, the electron beam suffers from both effects, requiring a selfconsistent treatment. In this study, we do not regularly distinguish the ion-trapping effect and the fast ion effect but treat the beam-ion interaction in a general sense. To clarify the beam-ion dynamics process and evaluate the beam characteristics, a numerical simulation code based on the "quasi-strong-strong" model has been developed, including modules for ionization, beam-ion interaction, synchrotron radiation damping, quantum excitation, bunch-by-bunch feedback, etc. The lattice of the High Energy Photon Source is used as an example to show the beam-ion instability and its mitigation. It is found that the beamion instability seriously occurs in a certain low beam current region. When the beam-ion instability is significantly driven and cannot be damped by the synchrotron radiation damping, a bunch-by-bunch feedback system based on a finite impulse response filter can be adopted to mitigate it effectively.
\end{abstract}

DOI: 10.1103/PhysRevAccelBeams.23.074401

\section{INTRODUCTION}

The beam-ion interaction, a two-stream effect coupled by the nonlinear Coulomb force, may pose an operation risk in high-intensity, low-emittance electron rings. This effect has been observed in many existing machines such as Advanced Light Source [1,2], Pohang Light Source [3], Shanghai Synchrotron Radiation Facility [4], Cornell Electron Storage Ring-Test Accelerator [5], Optimized Light Source of Intermediate Energy to LURE [6], etc. The accumulated ions, derived from ionization between electrons and residual gas molecules, interact with the electron beam particles resonantly and cause coherent and incoherent electron beam deformation, such as beam centroid oscillation, rms beam size and emittance growth, energy spread blowup, and even beam losses.

In previous studies, the beam-ion effect [7-9] is divided into two circumstances known as the ion-trapping effect and fast ion effect. In the ion-trapping study, the trapping condition [8] is

$$
A>A_{\mathrm{th}}=\frac{N_{b} r_{p} \Delta T_{b} c}{2 \sigma_{x, y}\left(\sigma_{x}+\sigma_{y}\right)},
$$

\footnotetext{
*lichao@ihep.ac.cn

Published by the American Physical Society under the terms of the Creative Commons Attribution 4.0 International license. Further distribution of this work must maintain attribution to the author(s) and the published article's title, journal citation, and DOI.
}

where $N_{b}$ is the bunch population, $r_{p}$ is the classical proton radius, $\Delta T_{b}$ is the bunch spacing, $c$ is the speed of light, and $\sigma_{x}$ and $\sigma_{y}$ are the rms beam sizes. Ions with a mass number $A$ larger than the threshold $A_{\text {th }}$ will be trapped transversely in the space charge potential well of the electron beam and interact with the electron beam particles resonantly, leading to beam performance deterioration or even beam losses. Ions with a mass number lower than $A_{\text {th }}$ will be overfocused by the bunched beam particles and hardly disturb the beam. The derivation of the ion-trapping condition is based on the assumption of linear space charge and an even beam filling pattern. $A_{\mathrm{th}}$ varies along the accelerator, since the betatron function varies. Results obtained from the ion trapping play a guiding role for vacuum selection and beam intensity uplimit prediction. To simplify the dynamics, the ion-trapping study usually assumes a steady state - the accumulated ions are saturated to an equilibrium state over thousands of turns. In this frame, a beam-ion eigensystem can be analytically established and used to predict the coupling resonance conditions [10]. Correspondingly, the fast ion effect focuses on the transient effect in one turn. The ions generated in the previous turn are cleaned up and do not disturb the beam performance in the following turns. In the timescale of one turn, the ions generated by the leading bunches oscillate transversely and disturb the motions of the subsequent bunches-a coupled bunch instability.

The ion-trapping effect and fast ion effect are both derived from the beam-ion Coulomb interaction but emphasize the dynamics process on different timescales. In the analytical studies, approximations adopted in the iontrapping effect and fast ion effect are always violated by 
constraints such as an uneven beam filling pattern, varying betatron functions, residual ions accumulated turn by turn, etc. It leads to an impossible task to build a general model for an analytical study. In this paper, our studies on the beam-ion effect are mainly focused on numerical simulations. A code based on the "quasi-strong-strong" approach is developed [11], in which the ion-trapping and fast ion effects are not distinguished and consistently treated. Modules of ionization, beam-ion interaction, synchrotron radiation damping, quantum excitation, and bunch-by-bunch feedback are also established. The lattice of the High Energy Photon Source (HEPS) [12], a ultralow-emittance ring under construction in Beijing, is adopted as an example to show the beam-ion effect in detail. It is found that the beam-ion interaction significantly affects the beam performance only when ions can be extensively accumulated in a certain current region. When the beam current goes higher in the operation mode, the ions will be overfocused, get lost in gaps between the neighboring bunches, and hardly affect the beam.

Generally, the methodologies to mitigate the beam-ion effect are (i) adjust the beam filling pattern by including enough empty buckets in the bunch train; (ii) get rid of ions with certain accelerator elements; and (iii) prevent the growth of the beam-ion instability by introducing a feedback system [13]. The first approach can extensively reduce the number of accumulated ions. In the empty bucket gaps, the trapped ions would drift to large amplitudes and get lost on the pipe or form a diffuse ion halo. However, this approach is a partial solution, since the electron bunches have been affected already. The beam deformation by the beam-ion interaction will accumulate, and a final influence could appear. The second and the third approaches both require extra hardware, which brings in new sources of lattice impedance. However, the bunch-by-bunch feedback system is a versatile [14] technique, as it can also suppress beam instabilities due to impedances.

This paper is organized as follows: In Sec. II, the physical process and models of beam-ion interaction will be discussed briefly. The logic flow and basic numerical simulation approaches used will be given. In Sec. III, both the "weak-strong" and quasi-strong-strong simulations of the beam-ion effect in HEPS will be discussed in detail. The bunch-by-bunch feedback will be discussed in Sec. IV. By introducing an appropriate feedback system, the beam-ion instability can be effectively suppressed. Discussion and conclusions are given in Sec. V.

\section{PHYSICS MODEL AND NUMERICAL APPROACHES}

\section{A. Basic model of beam-ion interaction}

Denoting $P$ and $T$ as the residual gas pressure and temperature, respectively, the molecule density $n_{i}$ is

$$
n_{i}=\frac{P N_{A}}{R T},
$$

where $R$ and $N_{A}$ are the ideal gas constant and Avogadro constant, respectively. Denoting $\sum$ as the ionization collision cross section and $N_{b}$ as the number of electron particles passing by, the number of ions generated due to beam-residual gas collision per unit length is

$$
\Lambda=\sum n_{i} N_{b} \text {. }
$$

For simplicity, we assume the beam-ion interaction is localized at the lumped interaction locations and affects the beam only transversely. In addition, ions generated do not move longitudinally. When the electron bunches pass through the interaction point one by one, new ions will be randomly generated within the sizes of the electron bunches. The interval times of new ion generation in bunch trains are decided by the beam filling pattern. The ions accumulated at the interaction point are kicked by the passing beam bunch and thereafter freely drift transversely until the next beam bunch comes. Some ions might get lost on the pipe during this process. Because of these ion generation and loss mechanisms, a final dynamical quasiequilibrium ion distribution can be foreseen.

Figure 1 shows the logic flow of the simulations. $S_{i}$ represents the lumped interaction point. When one electron bunch passes by, the transverse momentum and position of the accumulated ions are updated according to the time interval from the passing electron bunch to the next coming electron bunch. As to the bunched electrons, after the momentum kicks induced by the accumulated ions and taking into account the effects of synchrotron radiation and quantum excitation, they are transferred to the next interaction point $S_{i+1}$ by applying a linear transport matrix $M\left(S_{i+1} \mid S_{i}\right)$.

\section{B. Coulomb interaction}

In general, at the interaction point, the motion of the $i$ th accumulated ion $\vec{X}_{i}$ and the $k$ th electron particle in the $j$ th bunch $\vec{x}_{k ; j}$ can be expressed as

$$
\begin{aligned}
\frac{d^{2} \vec{X}_{i}}{d t^{2}}+K_{i}(s) \vec{X}_{i}+\sum_{k=0}^{N_{j}} \vec{F}_{C}\left(\vec{X}_{i}-\vec{x}_{k ; j}\right) & =0, \\
\frac{d^{2} \vec{x}_{k ; j}}{d s^{2}}+K_{e}(s) \vec{x}_{k ; j}+\sum_{i=0}^{N_{i}} \vec{F}_{C}\left(\vec{x}_{k ; j}-\vec{X}_{i}\right) & =0,
\end{aligned}
$$

respectively, where $\vec{F}_{C}$ is the Coulomb force between the ions and electron particles, $K_{i}(s)$ and $K_{e}(s)$ represent the lattice focusing strength on ion and electron particles, respectively, and $N_{j}$ and $N_{i}$ represent the number of electrons in the $j$ th bunch and the number of accumulated ions at the interaction point, respectively. In our model, the bunched beam is assumed to have a Gaussian distribution, and the field generated by the electron bunch at spatial 


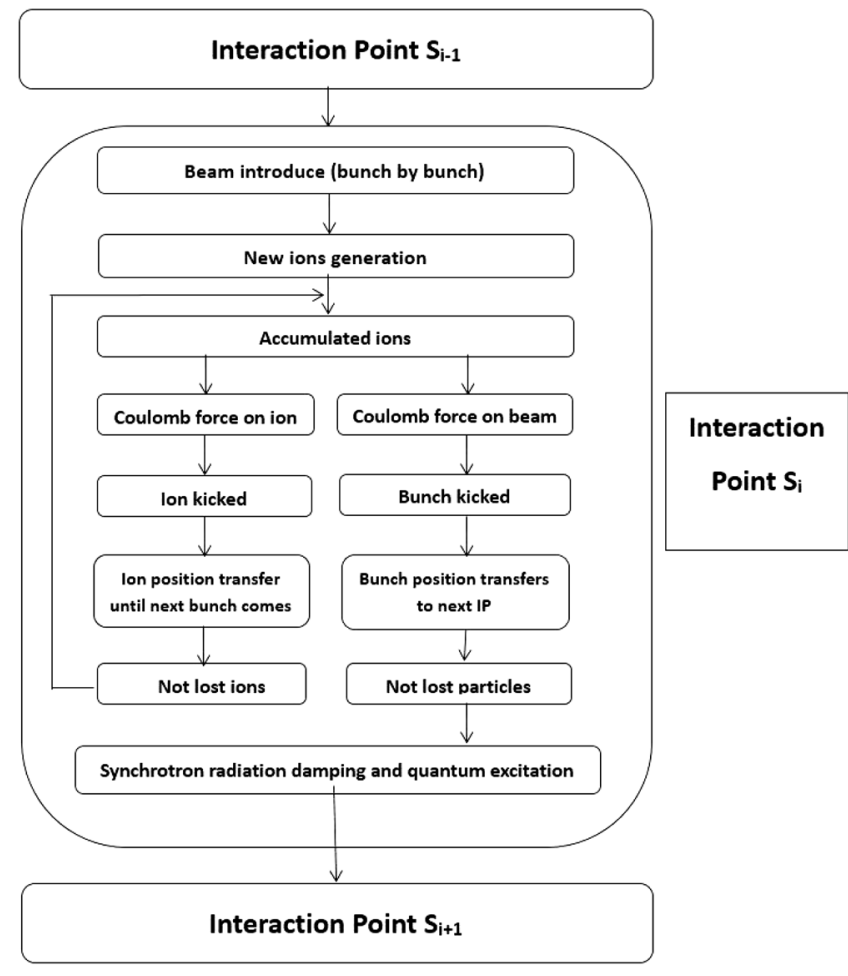

FIG. 1. Logic flow of the beam-ion interaction in simulation at the interaction points $S_{i}$. The ion generation, beam-ion interaction, beam and ion loss assertion, effects of synchrotron radiation, quantum excitation, and beam transportation are sequentially calculated.

location $(x, y)$, referenced to the bunch center at interaction $S_{i}$, is obtained with the 2D Bassetti-Erskine formula [15]:

$$
\begin{aligned}
E_{C, y}+i E_{C, x}= & \frac{n_{b}}{2 \epsilon_{0} \sqrt{2 \pi\left(\sigma_{x}^{2}-\sigma_{y}^{2}\right)}}\left\{w\left(\frac{x+i y}{\sqrt{2\left(\sigma_{x}^{2}-\sigma_{y}^{2}\right)}}\right)\right. \\
& -\exp \left(-\frac{x^{2}}{2 \sigma_{x}^{2}}-\frac{y^{2}}{2 \sigma_{y}^{2}}\right) \\
& \left.+w\left(\frac{x \frac{\sigma_{y}}{\sigma_{x}}+i y \frac{\sigma_{x}}{\sigma_{y}}}{\sqrt{2\left(\sigma_{x}^{2}-\sigma_{y}^{2}\right)}}\right)\right\} \delta\left(S_{i}\right),
\end{aligned}
$$

where $n_{b}$ is the line density of the electron beam, $w(z)$ is the complex error function, and $i$ is the complex unit. Substituting Eq. (5) into Eq. (4), the explicit momentum kick on ions is

$$
\Delta p_{i, y}+i \Delta p_{i, x}=\frac{2 n_{b} r_{e} m_{e} c}{\gamma_{e}}\left(E_{C, y}+i E_{C, x}\right)
$$

where $r_{e}$ is the classical electron radius, $m_{e}$ is the electron mass, and $\gamma_{e}$ is the relativistic factor of the electron beam. The accumulated ion momentum change induced by the electron bunch passing by can be obtained by integrating Eq. (6) along the length of adjacent electron bunches.

As to the space charge potential well generated by the ions, since the ion distribution is usually not a Gaussian type, the Bassetti-Erskine formula is not directly suitable. A self-consistent particle-in-cell (PIC) [16] solver or ion density profile fitting [17] is needed to ensure a better resolution. In our model, a compromise approach is applied, called quasi-strong-strong. The ion distribution is truncated at 10 times the rms bunch size. The rms and centroid information of the truncated ion distribution are substituted in the Bassetti-Erskine formula to get the Coulomb potential produced by ions. Although this approach is not strict, it still shows the main features of the bunched beam and can explore this complex coupled dynamics in a reasonable computing time.

\section{Beam transportation}

We employ the accelerator coordinates $\boldsymbol{x}=\left(x, p_{x}, y, p_{y}\right.$, $\left.z, p_{z}\right)$ to describe the motion of electrons. Here $x, y$, and $z$ are horizontal, vertical, and longitudinal coordinates, respectively, while $p_{x}, p_{y}$, and $p_{z}$ are the corresponding momentum normalized by the total momentum of a reference particle. The electron beam transportation from interaction point to interaction point is handled in the $\boldsymbol{x}$ frame. As to the synchrotron radiation and quantum excitation effects, they have to be treated in a normalized coordinates $\boldsymbol{X}$ frame. Following Hirata's Beam-Beam interaction with a Crossing angle code [18], in general, the transportation consists of the following steps.

\section{From accelerator coordinates to normalized coordinates}

The transformation from accelerator variable $\boldsymbol{x}$ to normalized variable $\boldsymbol{X}$ can be written as

$$
\boldsymbol{X}=\boldsymbol{B R H} \boldsymbol{x}
$$

where $\boldsymbol{H}$ is the dispersion matrix characterized by the transverse dispersion functions $D_{x}, D_{p x}, D_{y}$, and $D_{p y} ; \boldsymbol{R}$ is the Teng matrix representing the coupling between the horizontal and vertical; and $\boldsymbol{B}$ represents the Twiss matrix. The specific forms of $\boldsymbol{H}, \boldsymbol{R}$, and $\boldsymbol{B}$ can be found in Ref. [19].

In the study of this paper, the beam-ion dynamics is limited to the transverse plane in the dispersion and coupling free region, so $\boldsymbol{H}$ and $\boldsymbol{R}$ are further degenerated to unit matrices.

\section{Synchrotron radiation and quantum excitation}

For the synchrotron radiation and quantum excitation effects, the transportation in the normalized coordinates is

$$
\left(\begin{array}{l}
\boldsymbol{X}_{\mathbf{1}} \\
\boldsymbol{X}_{\mathbf{2}}
\end{array}\right)=\lambda_{x}\left(\begin{array}{l}
\boldsymbol{X}_{\mathbf{1}} \\
\boldsymbol{X}_{\mathbf{2}}
\end{array}\right)+\sqrt{\epsilon_{x}\left(1-\lambda_{x}^{2}\right)}\left(\begin{array}{l}
\hat{\boldsymbol{r}}_{\mathbf{1}} \\
\hat{\boldsymbol{r}}_{\mathbf{2}}
\end{array}\right),
$$




$$
\left(\begin{array}{l}
\boldsymbol{X}_{\mathbf{3}} \\
\boldsymbol{X}_{\mathbf{4}}
\end{array}\right)=\lambda_{y}\left(\begin{array}{l}
\boldsymbol{X}_{\mathbf{3}} \\
\boldsymbol{X}_{\mathbf{4}}
\end{array}\right)+\sqrt{\epsilon_{y}\left(1-\lambda_{y}^{2}\right)}\left(\begin{array}{l}
\hat{\boldsymbol{r}}_{\mathbf{3}} \\
\hat{\boldsymbol{r}}_{\mathbf{4}}
\end{array}\right)
$$

Here $\hat{r}$ 's are independent Gaussian random variables with unit variance; $\lambda_{x(y)}=\exp \left(-1 / T_{x(y)}\right)$ is the transport coefficient with $T_{x,(y)}$ representing the synchrotron radiation damping time in the units of number of turns; $\epsilon_{x}$ and $\epsilon_{y}$ are the normalized beam emittance in the horizontal and vertical direction, respectively.

\section{From normalized coordinates to accelerator coordinates}

Intuitively, the coordinate transformation from normalized variable $\boldsymbol{X}$ to accelerator variable $\boldsymbol{x}$ is

$$
\boldsymbol{x}=\boldsymbol{H}^{-1} \boldsymbol{R}^{-1} \boldsymbol{B}^{-1} \boldsymbol{X} .
$$

\section{Beam transportation from interaction point $S_{i}$ to $S_{i+1}$}

The electron beam transport from interaction point to interaction point is assumed to be a linear map:

$$
\boldsymbol{x}\left(\boldsymbol{S}_{i+\mathbf{1}}\right)=M\left(S_{i+1} \mid S_{i}\right) \boldsymbol{x}\left(S_{i}\right) .
$$

\section{Finite impulse response filter and bunch-by-bunch feedback}

Bunch-by-bunch feedback based on the finite impulse response (FIR) filter is an effective way to cure the coupled bunch instability. It detects transverse or longitudinal centroid positions of beam bunches, processes the position data to create kicker signals, and adds transverse or longitudinal kicks to the beam particles to damp their oscillations. Equation (12) gives the general form of the FIR filter in the time domain:

$$
\Theta_{n}=\sum_{k=0}^{N} a_{k} \theta_{n-k},
$$

where $a_{k}$ represents the filter coefficient and $\theta_{n-k}$ and $\Theta_{n}$ are the input and output, respectively, of the filter. The number of the input data $N+1$ is defined as taps of the filter.

With the FIR filter, the collective beam momentum change at the kicker in the $n$th turn due to the bunchby-bunch feedback is modeled as

$$
\begin{aligned}
& \delta \bar{x}_{x, n}^{\prime}=K_{x} \sum_{k=0}^{N} a_{k, x} \bar{x}_{n-k}, \\
& \delta \bar{y}_{y, n}^{\prime}=K_{y} \sum_{k=0}^{N} a_{k, y} \bar{y}_{n-k},
\end{aligned}
$$

where $\bar{x}_{n-k}$ and $\bar{y}_{n-k}$ are the bunch centroids of the $k$ th previous turn at the pickup; the kicker coefficients $K_{x}$ and $K_{y}$ are limited by the power constraints on the kicker. Each individual electron particle in the same bunch feels the same momentum kick at the kicker.

\section{SIMULATION STUDY OF BEAM-ION INTERACTION IN HEPS WITHOUT FEEDBACK}

HEPS is a $1.3 \mathrm{~km}$ ultralow-emittance electron storage photon source being built in Beijing, China. The main parameters of the HEPS lattice are listed in Table I. Carbon monoxide $(\mathrm{CO})$ with a temperature of $300 \mathrm{~K}$ and pressure of 1 nTorr is assumed as the main leaked gas. At the beam operation stage with $200 \mathrm{~mA}$ beam current, adopting the average betatron function and even filling pattern assumption, the critical mass number of the ion-trapping condition is estimated $A_{\text {th }} \approx 120$ by Eq. (1), indicating that the $200 \mathrm{~mA}$ electron beam hardly can be affected by the ionized CO. In the following study, the electron beam current $10 \mathrm{~mA}\left(A_{\mathrm{th}} \approx 10\right)$ is adopted to evaluate the beamion effect, since it gives the most serious effect as explained below. With the simplest case to explore the underlying physics, one beam-ion interaction point is set per turn. However, the setting of multi-interaction points must be checked in future studies. With the parameters shown in Table I, the time interval between the adjacent buckets can be obtained, that is $6 \mathrm{~ns}$. The beam filling pattern is one continuous bunch train of 680 electron bunches followed by 76 empty bunch bucket gaps.

\section{A. Weak-strong simulation}

Setting one macroelectron particle per bunch, the simulation automatically degenerates to the weak-strong case. Since the electron beam has smaller beam emittance and rms size in the vertical direction, a stronger beam-ion interaction in the vertical direction can be foreseen. Following the approaches used in previous studies $[9,20]$, the maximum

TABLE I. HEPS lattice parameters.

\begin{tabular}{lc}
\hline \hline Parameters & Values \\
\hline Energy & $6 \mathrm{GeV}$ \\
Circumference & $1360.4 \mathrm{~m}$ \\
Nominal emittance & $34.2 \mathrm{pm}$ \\
Working points & $114.14 / 106.23$ \\
Number of superperiods & 24 \\
Average betatron function & $4.5 / 8.1 \mathrm{~m}$ \\
Number of rf buckets & 756 \\
Beam current & $200 \mathrm{~mA}$ \\
SR damping time $(x / y)$ & $2386 / 4536$ turns \\
rms beam size $(x / y)$ & $12.4 / 5.26 \mu \mathrm{m}$ \\
Ion species & $\mathrm{CO}$ \\
Gas pressure & $1 \mathrm{nTorr}$ \\
Gas temperature & $300 \mathrm{~K}$ \\
\hline \hline
\end{tabular}




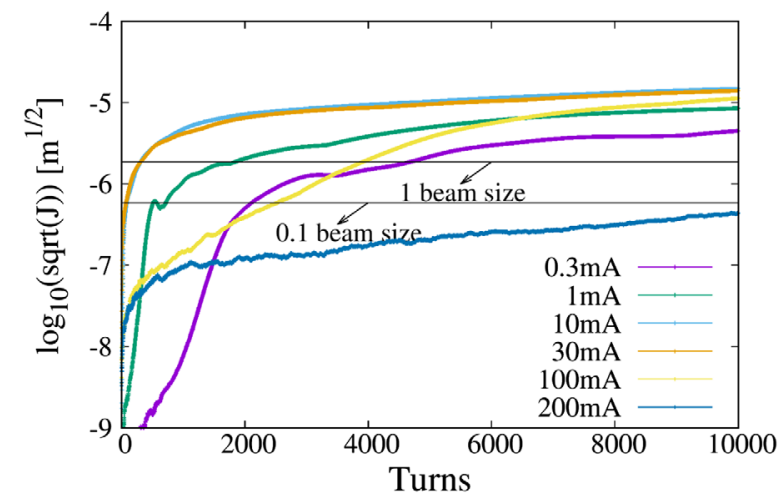

(a)

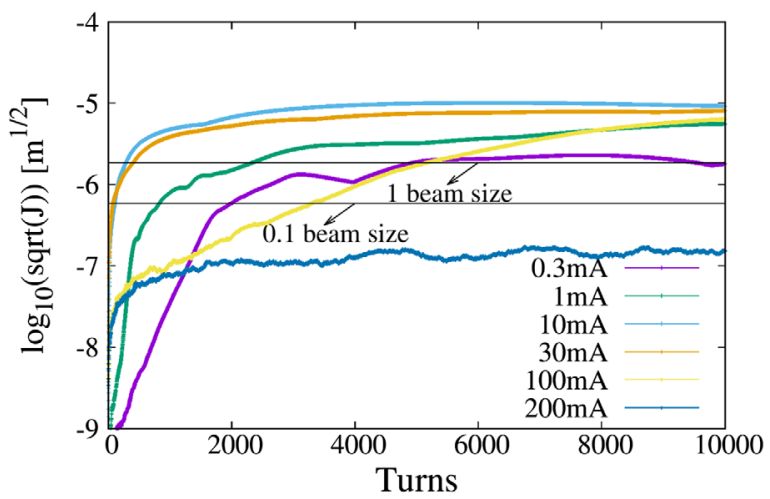

(b)

FIG. 2. The maximum bunch action as a function of the tracking turns in the weak-strong simulation without (left) and with (right) synchrotron radiation damping and quantum excitation. In each subfigure, there are six curves related to beam current settings $0.3,1,10$, 30, 100, and $200 \mathrm{~mA}$. The synchrotron radiation damping is not able to mitigate the beam-ion instability.

bunch dipole moment is recorded turn by turn. The vertical amplitude of the bunch centroid oscillation is represented by half of the Courant-Snyder invariant, which is

$$
J_{y}=\frac{1}{2}\left(\frac{1+\alpha_{y}^{2}}{\beta_{y}} y^{2}+2 \alpha_{y} y y^{\prime}+\beta_{y} y^{\prime 2}\right) \text {, }
$$

where $\alpha_{y}$ and $\beta_{y}$ are the local Twiss parameters.

Figure 2 shows the maximum bunch action $\sqrt{J_{y}}$ as a function of the tracking turns in the weak-strong simulation. The subfigures at left and right correspond, respectively, to the simulation without and with synchrotron radiation damping and quantum excitation. In each subfigure, there are six curves related to beam currents $0.3,1$, 10, 30, 100, and $200 \mathrm{~mA}$. For each beam current, the maximum square of bunch action $\sqrt{J_{y}}$ undergoes a sustained increase due to the beam-ion interaction and finally arrives to a stable value after 10000 turns evolution. The final values of $\sqrt{J_{y}}$ of the different cases increase with the beam current, reach a maximum value at $10 \mathrm{~mA}$, and then decrease. The beam is still significantly affected by the ions until $100 \mathrm{~mA}$. Up to $200 \mathrm{~mA}$, the trapping condition cannot hold; the ions are overfocused and mostly get lost in the empty gaps between the neighboring bunches where the beam centroid oscillation is below $0.1 \mathrm{rms}$ beam size. Comparing the results with and without synchrotron radiation damping and quantum excitation, the final $\sqrt{J_{y}}$ become smaller and get into an equilibrium state sooner when synchrotron radiation damping and quantum excitation are turned on.

It is noteworthy that the spontaneous synchrotron radiation damping in HEPS cannot stabilize the beam-ion instability except when the ions are overfocused in the $200 \mathrm{~mA}$ current case. In the following discussion, if not stated, $10 \mathrm{~mA}$ beam current is chosen as a default case to show the characteristics of the beam-ion interaction.
Figure 3 illustrates the bunch centroid oscillation and its coupled mode spectrum when synchrotron radiation damping is turned on. The oscillations of different bunches show a clear coupled leading-trailing motion as expected. With the number of turns increasing, more and more bunches at the tail part start to oscillate, which is one of the typical characteristics of the beam-ion instability. The unstable modes form resonant bands, and the resonant peaks are located at 50 and 70 in the horizontal and vertical direction, respectively.

In the simulations, ion particles beyond either 10 times effective rms beam size or the pipe aperture, are marked as lost ones. Figure 4 shows the total accumulated ion charge as a function of the tracking turns in the weak-strong model, where figures at left and right correspond to results obtained, respectively, without and with synchrotron radiation damping and quantum excitation. The accumulated ion charge is a compromise between the ion generation rate and ion loss rate. The ion loss rate lies in the ion's accumulated transverse momentum due to the passed electron bunches. With a higher beam current, more ions can be generated; however, a stronger beam-ion interaction also enlarges the ion loss rate conversely. Compared to the case with synchrotron radiation damping and quantum excitation at $10 \mathrm{~mA}$ beam current, without synchrotron radiation damping and quantum excitation, a sustained increasing beam action leads to a larger average distance between the new generated ions and the centroids of coming bunches. Then, a larger beam-ion interaction distance leads to a weaker ion focusing force, and more ions can be accumulated.

Figure 5 shows the ion density profile variation at the 8000th, 8400th, and 8900th turns in the weak-strong simulation with synchrotron radiation damping and quantum excitation. The center of the density profile shows an oscillation due to the beam-ion interaction, and the oscillation amplitude is consistent with the maximum bunch oscillation in Figs. 2 and 3. 


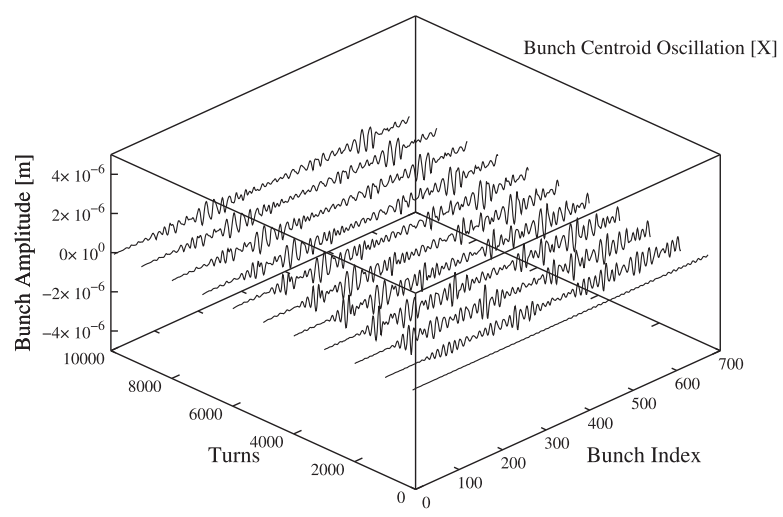

(a)

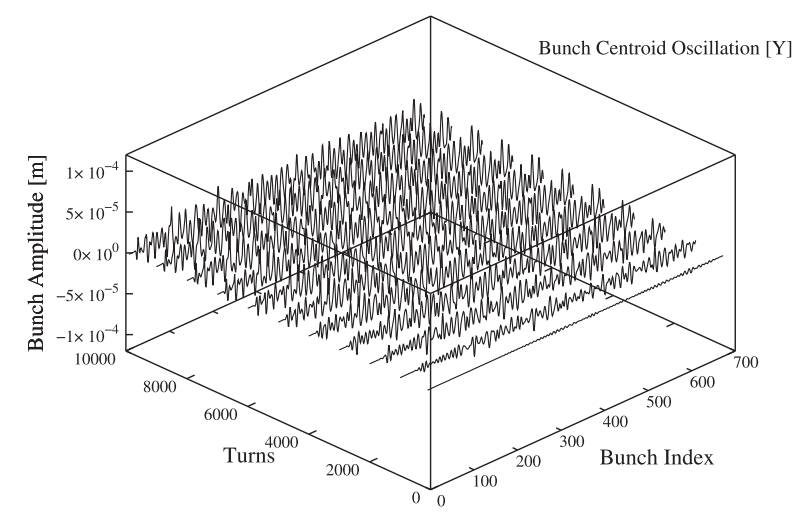

(c)

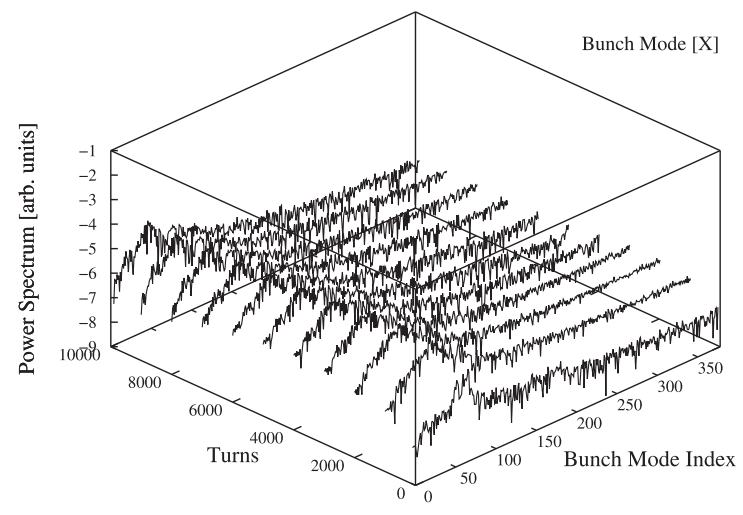

(b)

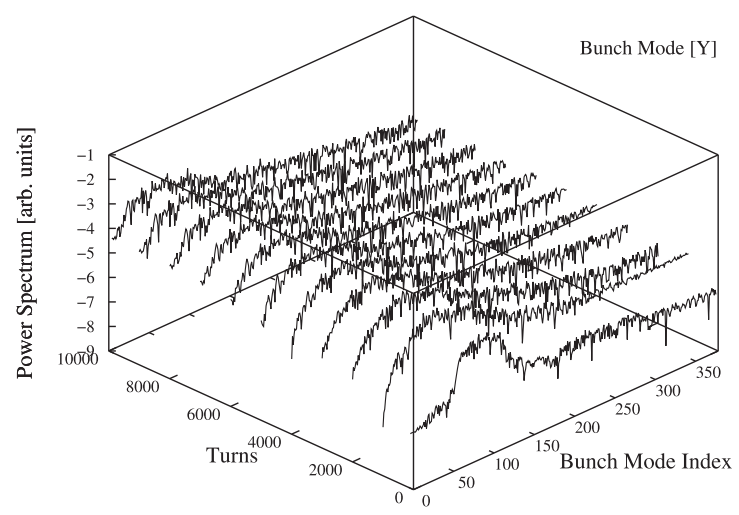

(d)

FIG. 3. Beam bunch oscillations in the horizontal (top) and vertical (bottom) and their power spectrum as a function of the passing turns. The coupled "leading-trailing" motions can be identified clearly. The simulation results are given by the weak-strong model with synchrotron radiation damping and quantum excitation.

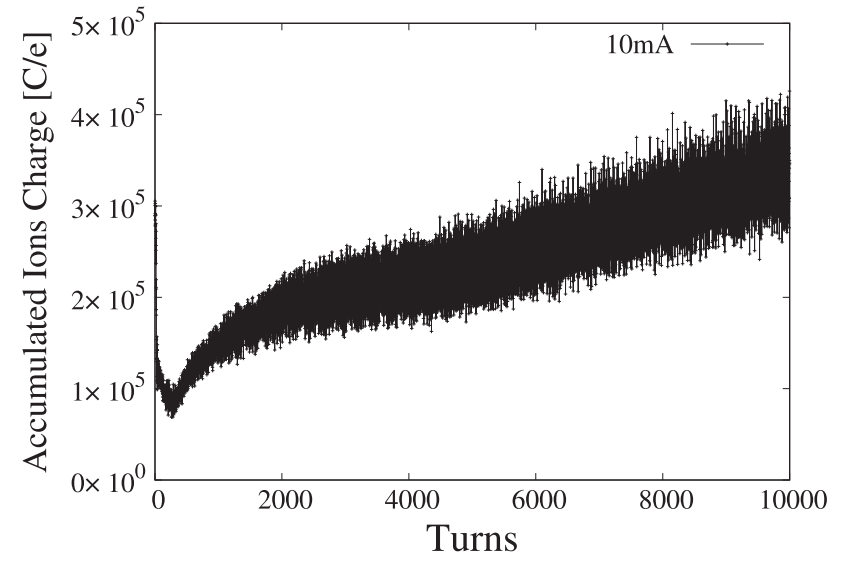

(a)

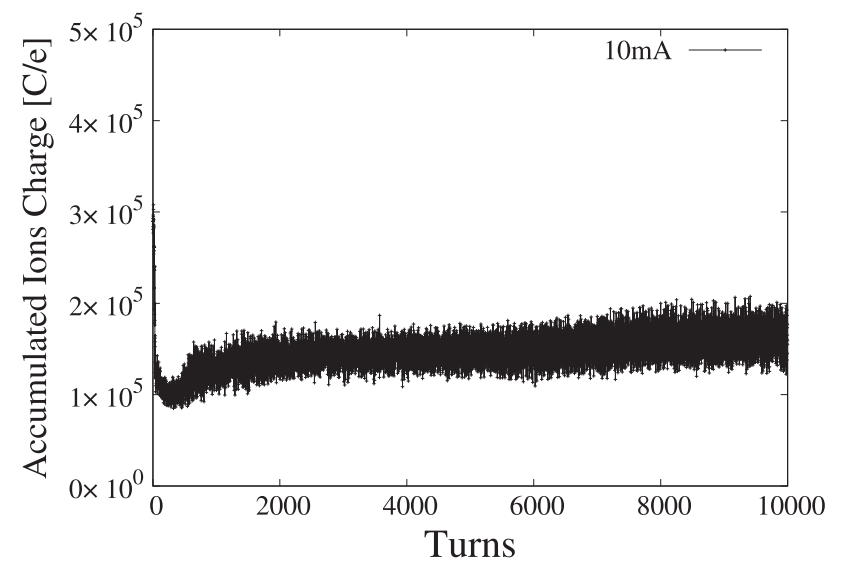

(b)

FIG. 4. The total ion charge accumulated at the interaction point as a function of the tracking turns in the weak-strong simulation without (left) and with (right) synchrotron radiation damping and quantum excitation.

\section{B. Quasi-strong-strong simulation}

To study the growth of beam effective emittance, rms emittance referenced to the ideal orbits, we will show the beam-ion dynamics with the quasi-strong-strong model in this subsection. The same beam and lattice conditions given in the last subsection are adopted in simulations. 


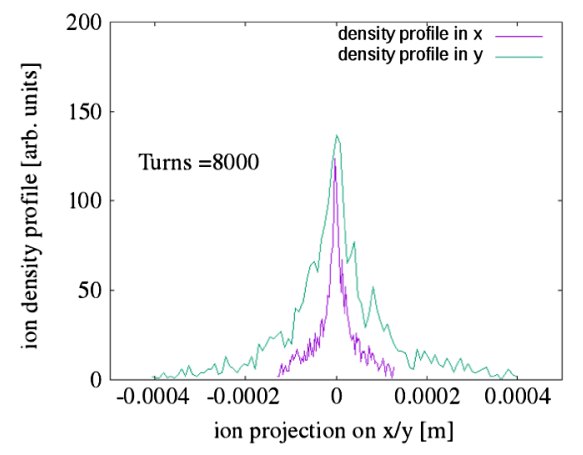

(a)

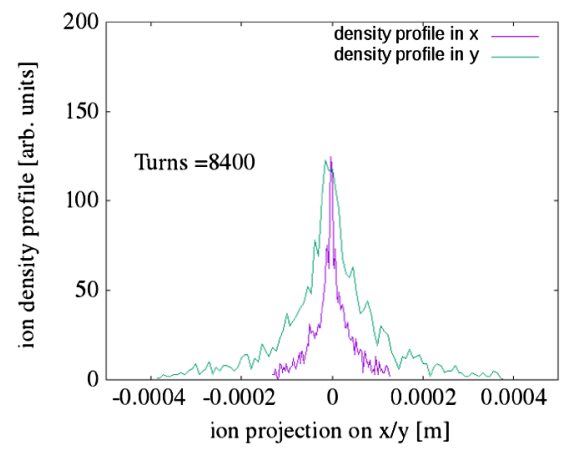

(b)

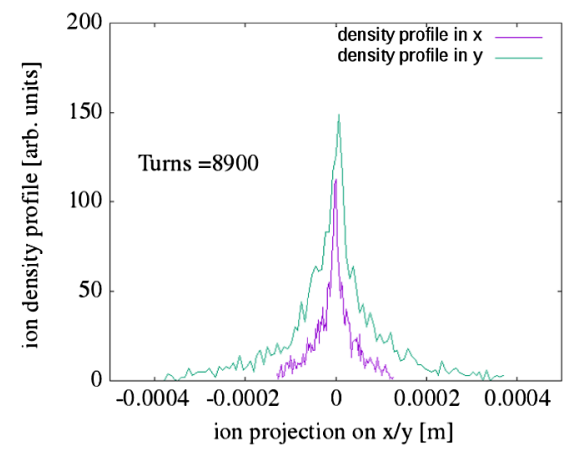

(c)

FIG. 5. Ion density profile sampled at the 8000th, 8400th, and 8900th turns (from left to right). The ion density profiles deviate from the Gaussian type and form a higher-density peak in the center. The density profile in the vertical direction shows an oscillation due to the beam-ion interaction. The simulation is launched with the weak-strong model and takes synchrotron radiation damping and quantum excitation into account.

Figure 6 shows the bunch oscillations and spectrum modes in the horizontal and vertical directions when the synchrotron radiation damping and quantum excitation are turned on. The maximum bunch centroid oscillation

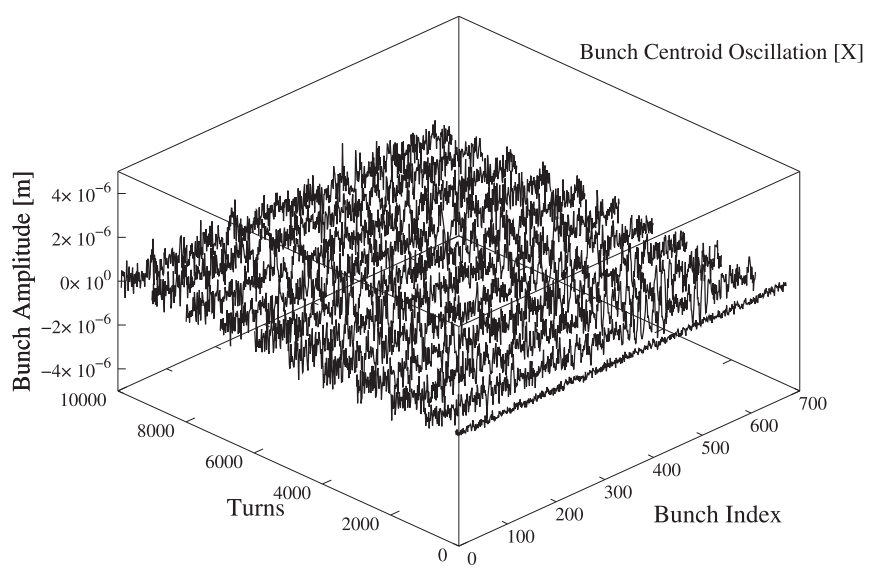

(a)

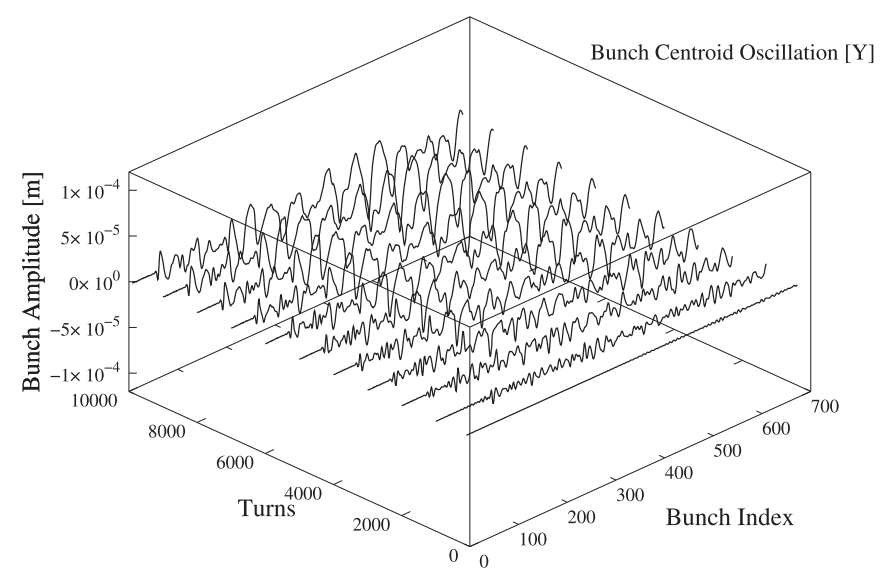

(c) amplitudes after 10000 turns are roughly 0.1 and 10 times rms beam size in the horizontal and vertical direction, respectively, which are consistent with the weak-strong results shown in Fig. 3. Since the noise from the limited

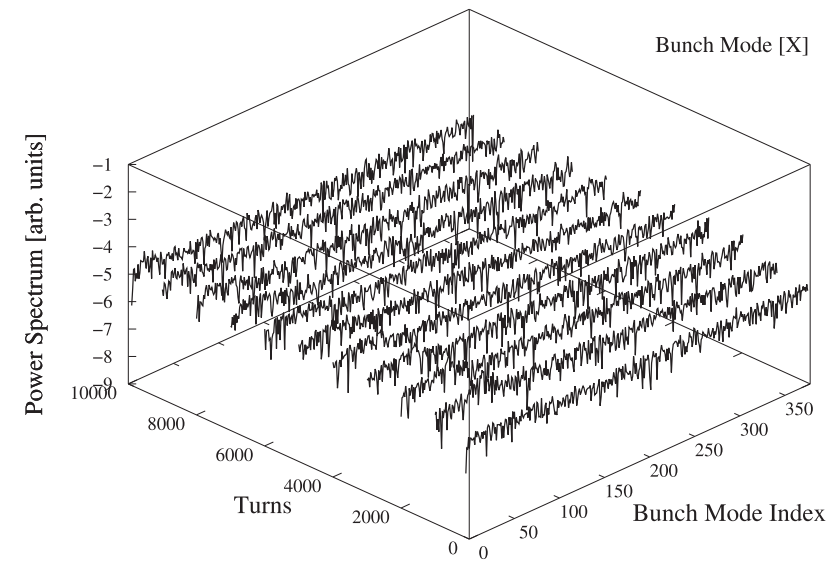

(b)

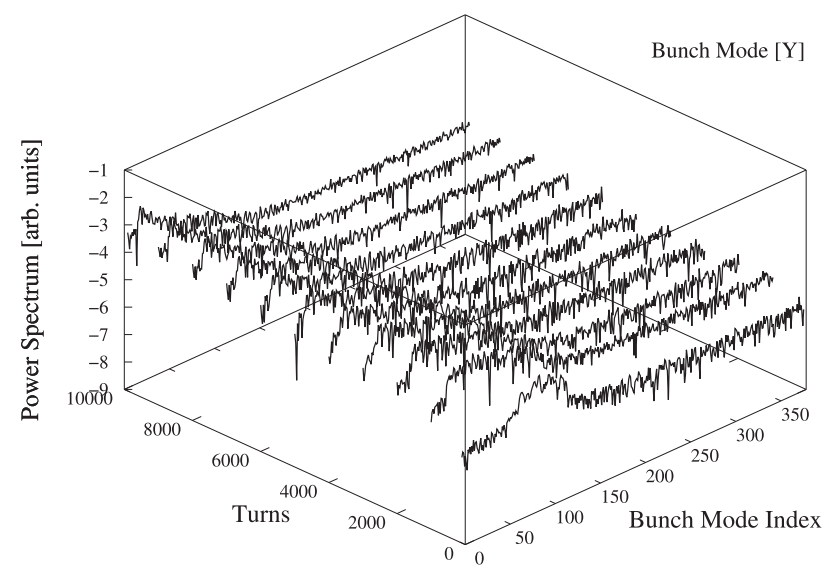

(d)

FIG. 6. Beam bunch oscillations in the horizontal (top) and vertical (bottom) and their frequency spectrum as a function of the passing turns. The simulation results are given by the "strong-strong" model with synchrotron radiation damping and quantum excitation. 


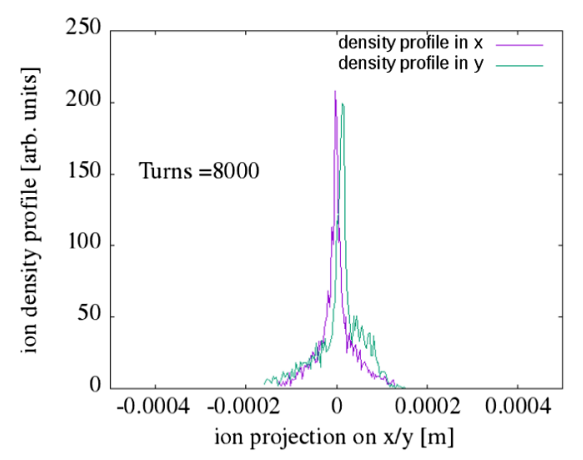

(a)

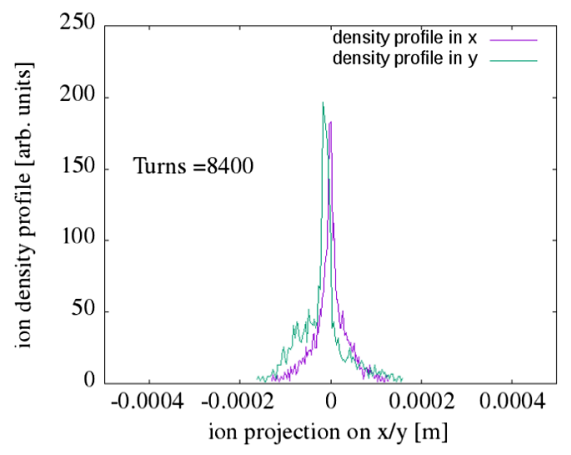

(b)

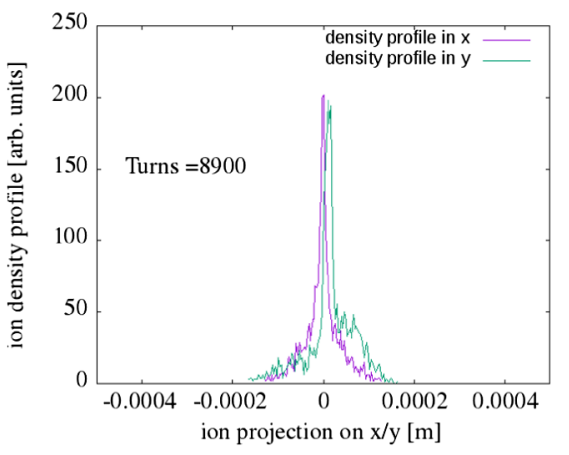

(c)

FIG. 7. Ion density profile sampled at the 8000th, 8400th, and 8900th turns (from left to right). The ion density profiles deviate from the Gaussian type and form a higher-density peak in the center. The density profile in the vertical direction shows an oscillation due to the beam-ion interaction. The simulation is launched with strong-strong model and takes synchrotron radiation damping and quantum excitation into account.

number of macroparticles cannot be ignored, especially with a small center oscillating amplitude in the horizontal direction, the bunch centroid oscillation within $0.1 \mathrm{rms}$ bunch size does not show a clear oscillation pattern. The corresponding mode spectrum does not show resonance peaks. In the vertical direction, the bunch centroid oscillation pattern is much clearer, and the mode resonance bands are consistent with the results given by the weak-strong model.

Similar to Fig. 5, Fig. 7 shows the ion density profile variation at the 8000th, 8400th, and 8900th turns. The amplitude of the ion density profile oscillation due to the beam-ion interaction is consistent with results shown in Fig. 6. Figure 8 depicts an explicit comparison of the ion density profile in the vertical direction between the simulations and Gaussian fitting at the 8000th turn. The average value and rms value of the ion distribution at the vertical direction in real space, obtained in numerical simulation, are directly used as the expected value and variance value during the fitting process. In both the weak-strong and strong-strong cases, the ion density profiles deviate from the Gaussian type and form a higher-density peak in the center. This result supports the analytical assumptions on the ion cloud distribution study in Ref. [13].

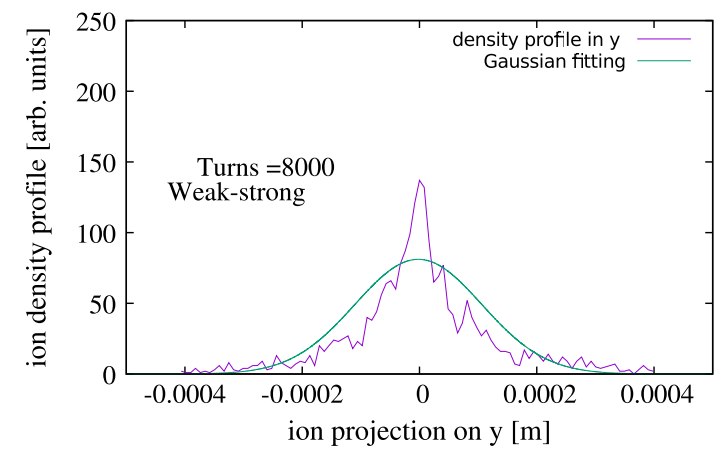

(a)
When one compares the results between the weak-strong and strong-strong cases, one thing that must be kept in mind is that the accumulated ions at the 8000th turns are different (Fig. 4). The variances used in Gaussian fitting are $1.08 \times 10^{-4}$ and $5.19 \times 10^{-5}$ for the weak-strong and strong-strong case, respectively, indicating a more concentrated profile center in the strong-strong simulation.

Figure 9 shows the accumulated ion charge and effective emittance variation as a function of the tracking turns. The final accumulated ion charge is roughly two-thirds that of the weak-strong case when the final "equilibrium" state is reached. The effective bunch emittance continuously increases in the vertical direction while being well maintained in the horizontal direction. In the following Sec. IV, a bunchby-bunch feedback system based on FIR filter techniques will be introduced to mitigate the beam-ion instability.

\section{A brief discussion}

In general, the strong-strong model includes ion and beam transverse oscillation frequency spreads that ease the beam-ion instability through Landau damping to some extent. However, in our case discussed above, the Landau

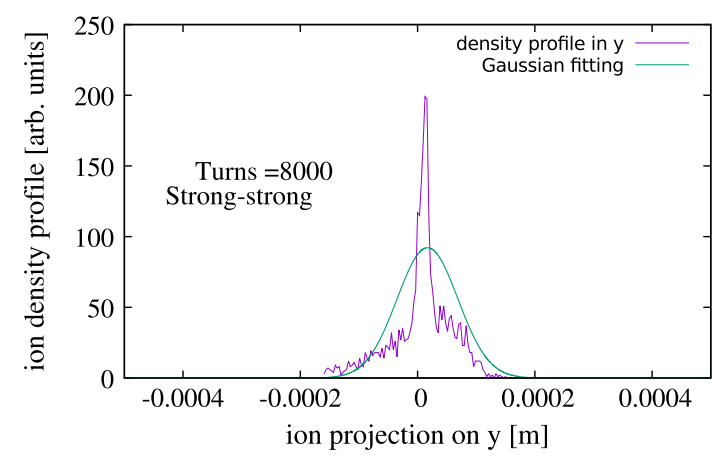

(b)

FIG. 8. The ion density profile comparison between simulations and Gaussian fitting in the vertical direction at the 8000th turn. Figures at left and right correspond to weak-strong and strong-strong simulations, respectively. 


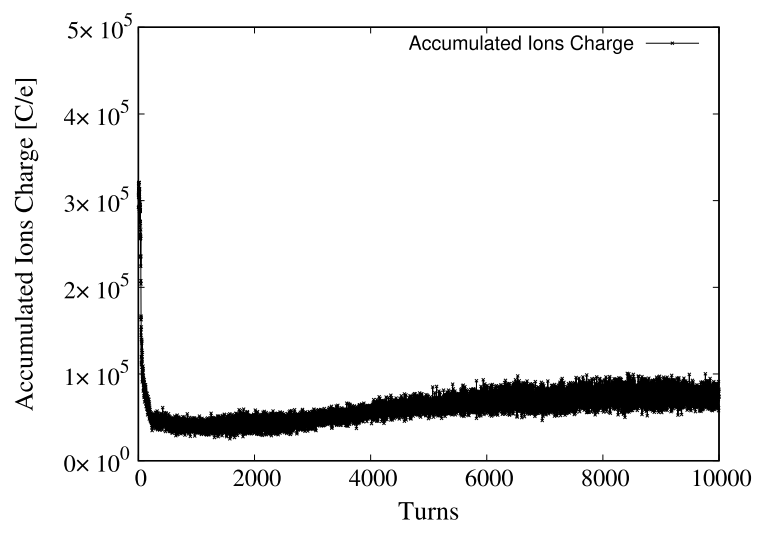

(a)

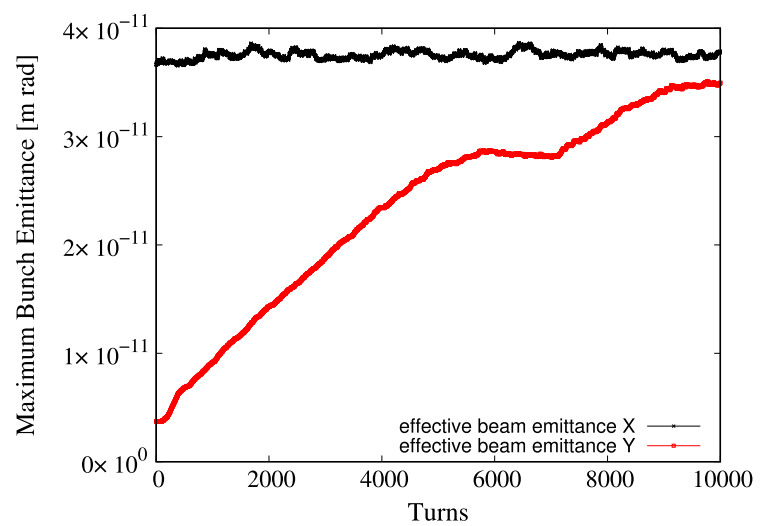

(b)

FIG. 9. Accumulated ion charge (a) and the maximum bunch emittance referenced to the ideal orbit (b) as functions of the passing turns; synchrotron radiation damping and quantum excitation are taken into account. The beam current is $10 \mathrm{~mA}$, and the simulation results are given by the quasi-strong-strong model.

damping mechanism does not play an obvious role. This is mainly due to the fact that the electron beam size is roughly 2 times smaller than that of the accumulated ions, so the electrons feel nearly linear space charge force from the accumulated ions. The effective rms emittance growth in the strong-strong simulation is mainly due to the centroid offset oscillation and only a little from beam phase space distortion and filamentation. The differences between the Gaussian profile assumption and the real beam (or ion) distributions must be noted. To get a more self-consistent process and better accuracy, a Poisson solver is required in further study.

From the analytical study point of view, several approaches have to be adopted to simplify the dynamics system, such as the smooth approximation to get a time-independent focusing lattice; the equilibrium state assumption of the accumulated ions-constant ion charge; the linear space charge approximation, even filling pattern, etc. More detailed information can be found in Refs. [7,8,21] in detail. Since the frequency of the bunch train also influences the ion frequency, here we use Eq. (8) from Ref. [13] to estimate the ion oscillation frequency:

$$
\begin{aligned}
\omega_{i, x(y)}^{2} & =\frac{2 \lambda_{e} r_{p} c^{2}}{A} \frac{1}{G_{x(y)} \sigma_{x(y)}\left(\sigma_{x}+\sigma_{y}\right)}, \\
G_{x(y)} & =\frac{\Sigma_{x(y)}\left(\Sigma_{x}+\Sigma_{y}\right)}{\sigma_{x(y)}\left(\sigma_{x}+\sigma_{y}\right)}, \\
\Sigma_{x(y)} & =\sqrt{\sigma_{x(y)}^{2}+\sigma_{i, x(y)}^{2}},
\end{aligned}
$$

where $\lambda_{e}$ is the electron line density and $\sigma_{i, x(y)}$ is the rms size of the ion cloud. Two cases with zero and equilibrium $\sigma_{i, x(y)}$ predict the ion oscillation frequency ranges, which are in the mode index interval $(17,73)$ and $(24,113)$ for the horizontal and vertical direction, respectively. Since the filling pattern is not even in our case, the frequency bands would shift to the lower region a little bit. Compared with the mode analysis in Figs. 3 and 6, where the resonance peak locates at 50 (70) in the horizontal (vertical), the model Eq. (15) gives quite reasonable predictions.

\section{BUNCH-BY-BUNCH FEEDBACK AND ITS INFLUENCE ON BEAM PERFORMANCE}

In general, the spectrum of the coupled bunch mode is

$$
\text { Spectrum } \propto M \omega_{0} \delta\left(\omega-\omega_{\beta}-p M \omega_{0}-\mu \omega_{0}\right),
$$

where $\omega$ is the bunch oscillation frequency, $M$ is the harmonic number, $p$ refers to any integer, $\mu$ is the mode index, and $\omega_{0}$ is the revolution frequency [22]. A welldesigned FIR filter, with direct current (dc) rejection, has zero amplitude response at a frequency of multiples of $\omega_{0}$, which indicates that the response at the fraction of the betatron tune $\omega_{\beta} / \omega_{0}$ is of the most importance in the FIR filter design.

Following the approaches used in Ref. [23], the time domain least square fitting method is adopted in this paper to get the filter coefficients $a_{k, x(y)}$ in Eq. (13). In the process of kicker strength calculation, we do not use the information of the bunch oscillation at the current turn that a oneturn delay condition $a_{0}=0$ is adopted. With this principle, two ten-tap FIR filters are designed. For clarity in simulation, the pickup and kicker are assumed to be located at the same place with zero dispersion, which means the phase responses of the filter at the target tunes is $-90^{\circ}$. Figure 10 shows the filter coefficients and the phase and amplitude responses as functions of the tune fraction. The first derivatives of the phase response curves at the target tune fractions are designed to be zero to enlarge the phase error tolerance. The stable working region is limited by the filter phase response curve within $(-180,0)^{\circ}$. The gain at the target tune fraction is at a local minimum and normalized 


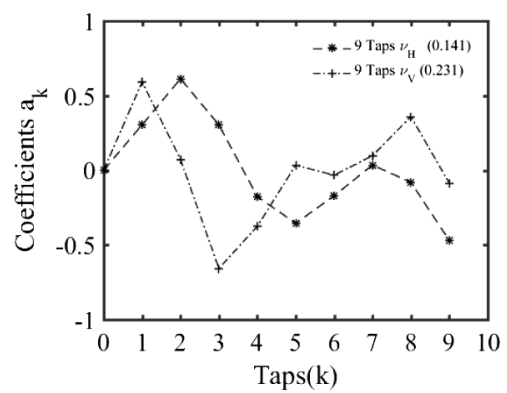

(a)

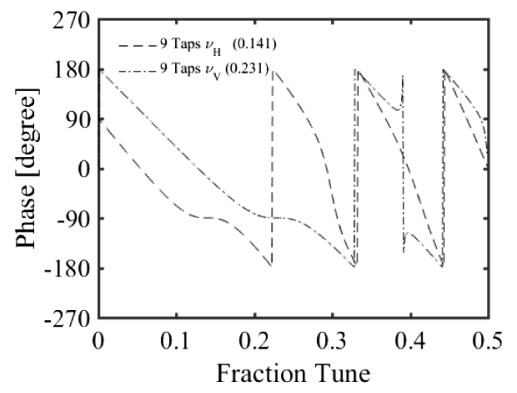

(b)

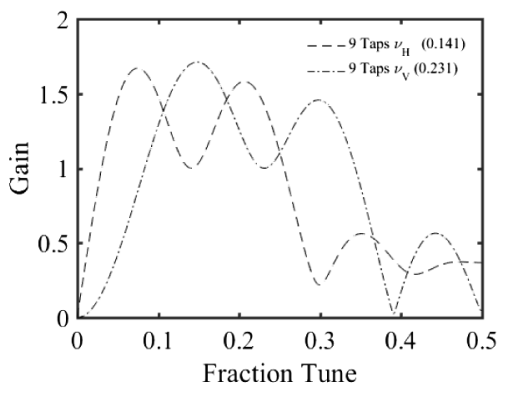

(c)

FIG. 10. FIR filter coefficients (a), frequency response of phase (b), and gain (amplitude) (c) of the nine-tap filters used in a bunch-bybunch feedback system. The horizontal and vertical target tune fractions are 0.141 and 0.231 , respectively.

to one. The dc components which are caused by the closed orbit distortions, unequal bunch signal shapes from pickup electrodes, and reflection at cable connections are rejected.

The shortest damping time $\tau_{F B}$ of the feedback can be approximated $[14,23]$ by

$$
\frac{1}{\tau_{F B}}=\frac{f_{r} \sqrt{\beta_{p} \beta_{k}}}{2 h E / e} \frac{\sqrt{2 P_{\max } R_{k}}}{\Delta X_{\max }}
$$

where $f_{r}$ is the revolution frequency, $h$ is the harmonic number, $\beta_{p}$ and $\beta_{k}$ are the betatron functions at the pickup and kicker, respectively, $E$ is the beam energy, $e$ is the electron unit charge, $\Delta X_{\max }$ is the maximum bunch oscillation that the feedback can suppress, $R_{k}$ is the kicker shunt impedance, and $P_{\max }$ is the maximum kicker power available. In HEPS, the shunt impedance at the kicker is $R_{k}=$ $123 \mathrm{~K} \Omega$, and the betatron function is $\beta_{p}=\beta_{k}=5 \mathrm{~m}$. Assuming that the power limit on the kicker is $P_{\max }=$ $1 \mathrm{KW}$ and the maximum bunch oscillation required to be damped $\Delta X_{\max }=0.1 \mathrm{~mm}$, the shortest damping time of the feedback can supply is roughly $60 \mu$ s.

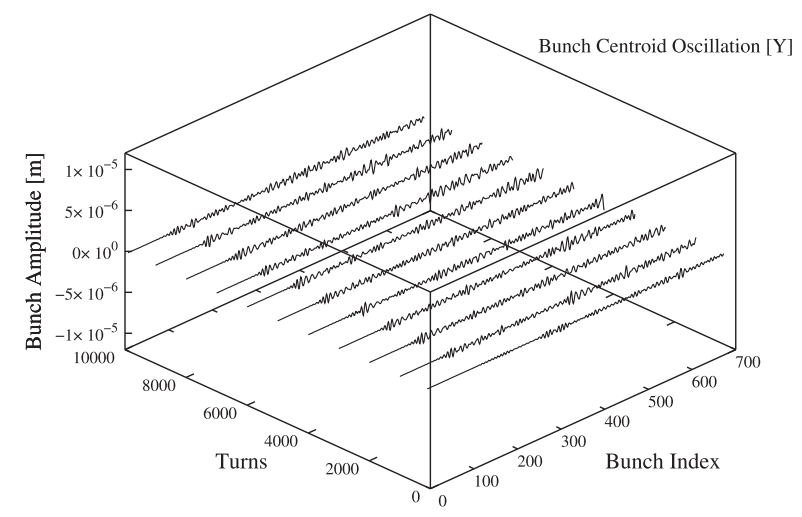

(a)
Figure 11 is calculated with the same parameters as those used in Fig. 3 except that the bunch-by-bunch feedback is turned on. Clearly, the bunch centroid oscillation amplitudes in the vertical direction are effectively damped down at least one order of magnitude smaller. Comparisons of the maximum beam size, bunch oscillations, and power spectrum with and without feedback are explicitly shown in Fig. 12. When the bunch-by-bunch feedback is turned on, the maximum bunch action is well maintained around $0.1 \mathrm{rms}$ beam size [Fig. 12(a)]; at the 5000th turn, the bunch oscillations due to beam-ion interaction are well eliminated [Fig. 12(b)]; correspondingly, the power spectrum of the bunch oscillation is roughly one order of magnitude smaller [Fig. 12(c)]. The flat top of the red curve in Fig. 12(c) is a direct effect of the bunch-by-bunch feedback system on the power spectrum. The position of the unstable bunch modes does not shift, since the intrinsic beam-ion interaction is not violated.

The simulation results given by the quasi-strongstrong model are shown in Fig. 13. The bunch centroid oscillation amplitudes are well damped down within $\pm 0.1 \mathrm{rms}$ beam size by the bunch-by-bunch feedback

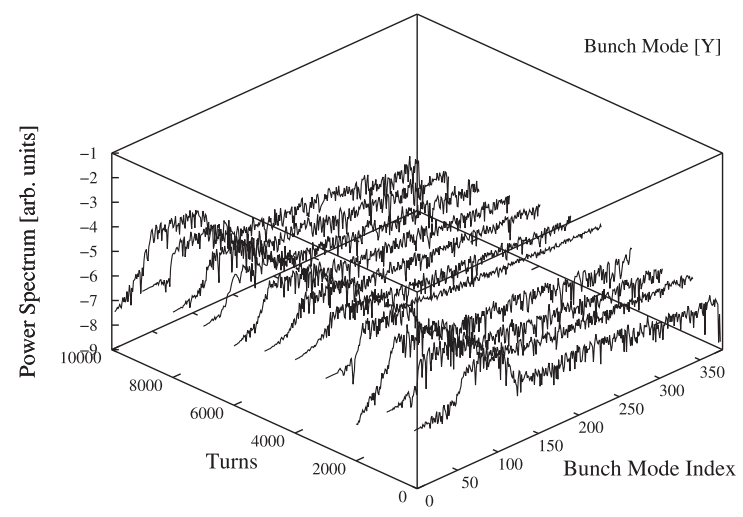

(b)

FIG. 11. Beam bunch oscillations in the vertical direction (left) and its frequency spectrum (right) as a function of the passing turns; both the synchrotron radiation damping and bunch-by-bunch FIR feedback system are taken into account. The beam current is $10 \mathrm{~mA}$, and the weak-strong model is used in the simulation. 


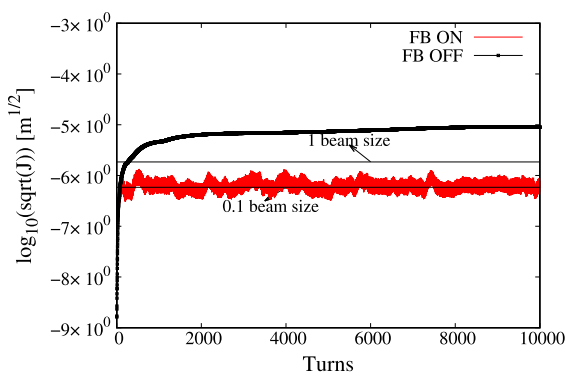

(a)

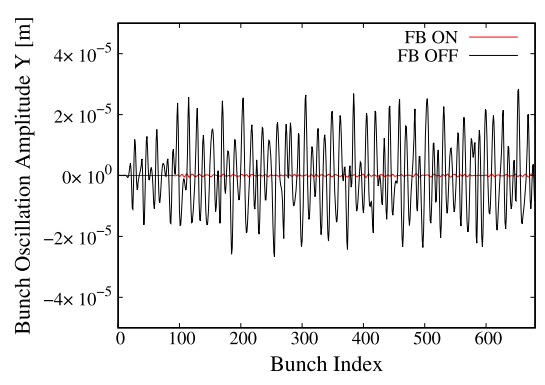

(b)

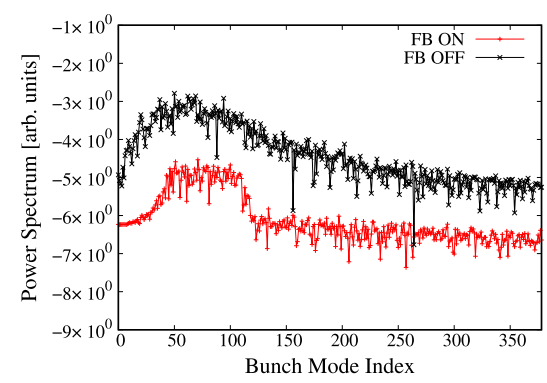

(c)

FIG. 12. The red and black curves show the maximum beam actions during 10000 turns evolution (a), the beam bunch oscillations at the 500th turn (b), and the related coupled bunch mode power spectrum (c) in the vertical plane with and without bunch-by-bunch feedback. The results are obtained from the weak-strong model taking synchrotron radiation damping and quantum excitation into account.

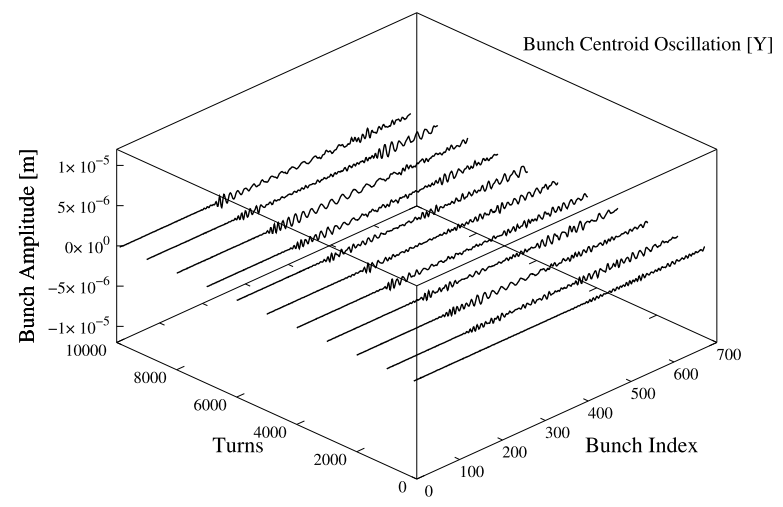

(a)

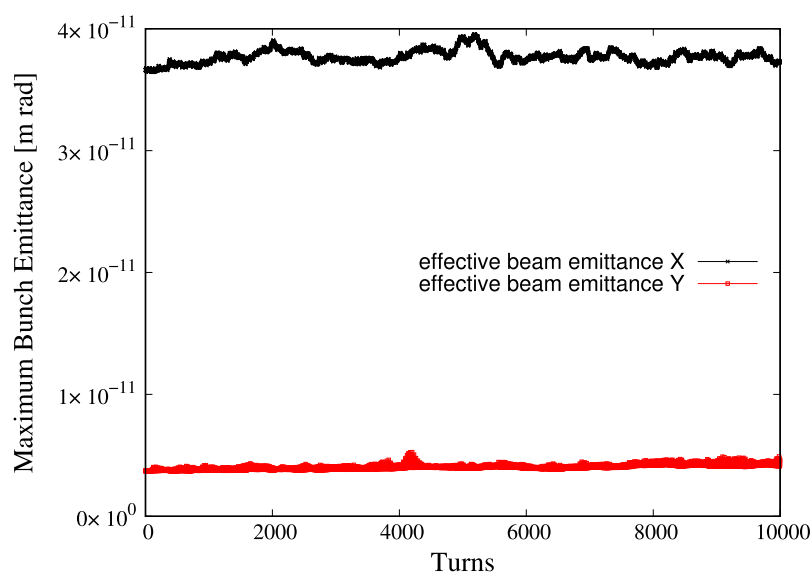

(b)

FIG. 13. Beam bunch oscillations (left) and the effective rms emittance (right) as functions of the passing turns; both the synchrotron radiation damping and bunch-by-bunch FIR feedback system are taken into account. The results are obtained from the quasi-strongstrong model.

system. The effective rms beam emittance is well maintained and shows only a tiny sustained increase due to the nonlinear Coulomb space-charge-induced phase space distortion and filamentations.

\section{CONCLUSIONS}

In this paper, we have discussed the beam-ion instability and its mitigation by the bunch-by-bunch feedback system. A simulation code is developed including modules for ionization, beam-ion interaction, synchrotron radiation damping, quantum excitation, and bunch-by-bunch feedback. The lattice parameters of HEPS are adopted to show the influence of the beam-ion effect. It is found that, in high-intensity and ultralow-emittance rings, the beam-ion instability is not a dominant mechanism when the beam current is high. It is because the ions generated are overfocused, get lost between the bunch gaps, and hardly disturb the beam. The beam-ion interaction significantly affects the beam performance only when ions can be extensively accumulated in a certain beam current region. If the beam-ion instability is too strong to be suppressed by synchrotron radiation damping, the bunch-by-bunch feedback based on the FIR filter technique is proved to be an effective approach to rescue the beam performance.

Studies in this paper can be improved to further steps in future studies. One point is adding a PIC solver into the code, since it is important to ensure a self-consistent process. Another point is related to the collective effect due to the impedance. It will be very interesting to know how the beam-ion interaction and the impedance interplay with each other. In the future, modules to deal with the impedance and wakefield will be developed to study the complex beam dynamics system, and then the ability of feedback system will be reevaluated.

\section{ACKNOWLEDGMENTS}

This work is supported by the Key Research Program of Frontier Sciences Chinese Academy of Sciences 
(QYZDJ-SSW-SLH001) and National Science Foundation of China $(11775239,11805217)$. Special thanks to Professor Takeshi Nakamura in Synchrotron Radiation Research Institute in Japan for his helpful discussion on FIR filters.

[1] F. Zimmermann, A. Chao, S. Heifets, M. Minty, J. Seeman, G. Stupakov, J. Byrd, J. Thomson, and T. Raubenheimer, Experiments on the fast beam-ion instability at the ALS, Technical Report No. SLAC-PUB-7617, Stanford University, Stanford Linear Accelerator Center, CA, 1997.

[2] J. Byrd and A. Chao, First observations of a "Fast beam-ion instability”, Phys. Rev. Lett. 79, 79 (1997).

[3] M. Kwon, J. Y. Huang, T.-Y. Lee, I. S. Ko, Y. H. Chin, H. Fukuma, M. Isawa, K. Ohmi, and M. Tobiyama, Experimental results on the fast beam-ion instability, Phys. Rev. E 57, 6016 (1998).

[4] B. Jiang, G. Xia, L. Han, G. Liu, Z. Dai, and Z. Zhao, Investigation of fast ion instability in SSRF, Nucl. Instrum. Methods Phys. Res., Sect. A 614, 331 (2010).

[5] A. Chatterjee, K. Blaser, M. Ehrlichman, D. Rubin, and J. Shanks, Fast Ion Instability at CESR-TA, in Proceedings of the 5th International Particle Accelerator Conference (IPAC 2014), Dresden, Germany, 2014 (JACoW, Geneva, Switzerland, 2014), TUPRI036, https://doi.org/10.18429/ JACoW-IPAC2014-TUPRI036.

[6] R. Nagaoka, M. P. Level, L. Cassinari, M. E. Couprie, M. Labat, C. Mariette, A. Rodriguez, and R. Sreedharan, Beam instability observations and analysis at SOLEIL, in Proceedings of the 22nd Particle Accelerator Conference, PAC-2007, Albuquerque, NM (IEEE, New York, 2007), pp. 2019-2021.

[7] T. Raubenheimer and F. Zimmermann, Fast beam-ion instability. I. Linear theory and simulations, Phys. Rev. E 52, 5487 (1995).

[8] G. Stupakov, T. Raubenheimer, and F. Zimmermann, Fast beam-ion instability. II. Effect of ion decoherence, Phys. Rev. E 52, 5499 (1995).

[9] K. Ohmi, Numerical study for the two-beam instability due to ions in electron-storage rings, Phys. Rev. E 55, 7550 (1997).
[10] K. Ohmi, F. Zimmermann, and E. Perevedentsev, Wakefield and fast head-tail instability caused by an electron cloud, Phys. Rev. E 65, 016502 (2001).

[11] C. Li, https://github.com/ChaoLiIHEP/FASTION.

[12] Y. Jiao, G. Xu, X.-H. Cui, Z. Duan, Y.-Y. Guo, P. He, D.-H. Ji, J.-Y. Li, X.-Y. Li, C. Meng, Y.-M. Peng, S.-K. Tian, J.-Q. Wang, N. Wang, Y.-Y. Wei, H.-S. Xu, F. Yan, C.-H. Yu, Y.-L. Zhao, and Q. Qin, The HEPS project, J. Synchrotron Radiat. 25, 1611 (2018).

[13] L. Wang, Y. Cai, T. Raubenheimer, and H. Fukuma, Suppression of beam-ion instability in electron rings with multibunch train beam fillings, Phys. Rev. Accel. Beams 14, 084401 (2011).

[14] M. Lonza and H. Schmickler, Multi-bunch feedback systems, arXiv:1601.05258.

[15] M. Bassetti and G. A. Erskine, Closed expression for the electrical field of a two-dimensional Gaussian charge, Technical Report No. CERN-ISR-TH-80-06, CERN, Geneva, 1980.

[16] C. Li, Z.-L. Zhang, X. Qi, X.-B. Xu, Y. He, and L. Yang, Beam dynamics study of RFQ for CADS with a 3D space-charge-effect, Chin. Phys. C 38, 037005 (2014).

[17] https://ops.aps.anl.gov/manuals/elegant_latest/elegant.pdf.

[18] K. Hirata, BBC user's guide: A computer code for beambeam interaction with a crossing angle, CERN SL Note No. 97-57 AP, 1997.

[19] Y. Zhang, K. Ohmi, and L. Chen, Simulation study of beam-beam effects, Phys. Rev. Accel. Beams 8, 074402 (2005).

[20] G. Xia and E. Elsen, Simulation study of ion effect in the ILC electron damping ring, Nucl. Instrum. Methods Phys. Res., Sect. A 593, 183 (2008).

[21] E.-S. Kim and K. Ohmi, Simulations on the fast-ion instability in the international linear collider damping rings, Jpn. J. Appl. Phys. 48, 086501 (2009).

[22] A.W. Chao, Physics of Collective Beam Instabilities in High Energy Accelerators (Wiley, New York, 1993).

[23] T. Nakamura, Single-loop multi-dimensional digital feedback by FIR filters, http://acc-web.spring8.or.jp/ nakamura/ reports/Feedback_with_FIR_Filter_draft1.pdf. 